\title{
Equivalence of two different integral representations of droplet distribution moments in condensing flow
}

\author{
Rob Hagmeijer \\ Department of Mechanical Engineering, University of Twente, P.O. Box 217, 7500 AE Enschede, \\ The Netherlands
}

(Received 7 October 2002; accepted 8 October 2003; published online 5 December 2003)

\begin{abstract}
It is proved that two different and independently derived integral representations of droplet size distribution moments encountered in the literature are equivalent and, moreover, consistent with the general dynamic equation that governs the droplet size distribution function. One of these representations consists of an integral over the droplet radius while the other representation consists of an integral over time. The proof is based on analytical solution of the general dynamic equation in the absence of coagulation but in the presence of both growth and nucleation. The solution derived is explicit in the droplet radius, which is in contrast with the literature where solutions are presented along characteristics. This difference is essential for the equivalence proof. Both the case of nonconvected vapor as well as the case of convected vapor are presented. (C) 2004 American Institute of Physics. [DOI: 10.1063/1.1630052]
\end{abstract}

\section{INTRODUCTION}

Reported investigations with respect to nonequilibrium condensation without coagulation in transonic nozzle flows ${ }^{1-6}$ describe the liquid mass fraction, i.e., the ratio of liquid mass per unit volume to the mass of the liquid-vapor mixture per unit volume, in terms of an integral over time containing both the droplet production rate and the droplet growth rate in its integrand. In contrast, reported investigations with respect to aerosol dynamics describe the liquid mass fraction in terms of an integral over the droplet radius, see, e.g., Ref. 7, which contains the droplet size distribution function in its integrand. These two different formulations are not only observed for the liquid mass fraction that is related to the number of droplets and the mean droplet volume, but also for the mean droplet radius and mean droplet surface.

To the author's knowledge, the time integral and the radius integral, respectively, have been formulated independently. The time integral, on the one hand, has been derived from physical reasoning in a very direct way, without employing a partial differential equation. The radius integral, on the other hand, is actually a definition. This observation gives rise to the following question: can it be proved that both formulations are equivalent? It is our objective to provide such a proof.

Since the so-called general dynamic equation balances the time and radius derivatives of the droplet size distribution function with the nucleation and growth rates, it is natural that equivalence of the two different integral formulations be consistent with the general dynamic equation. We will show that this is indeed the case. At first sight, it may seem not too difficult a problem to provide the equivalence proof. We have found, however, that it is necessary to obtain the analytical solution of the general dynamic equation as an ex- plicit expression in terms of the droplet radius, which is not trivial.

The paper is organized in the following way. First, the investigated problem is formulated in detail and then it is solved for two different cases: nonconvected vapor (at rest) and convected vapor (a prescribed velocity field). The solution of the nonconvected vapor case is illustrative and contains all of the essential steps to be taken to solve the convected vapor case. The solution of the convected vapor case is slightly more complex and leads to detailed expressions for the time integral formulation frequently encountered in the literature.

\section{PROBLEM FORMULATION}

The composition of a condensing vapor at a certain time instant $t$ in terms of the number of droplets per unit volume and the distribution of these droplets over a range of radii is conveniently described by the radius distribution function $f(r, t)$. This function is defined such that for any given $r$ $>0$ and $t>0$, the number of droplets per unit volume with radii smaller than $r$ is given by

$$
N(r, t)=\int_{0}^{r} f\left(r^{\prime}, t\right) d r^{\prime} .
$$

As a consequence the total number of droplets per unit volume at time $t$, denoted by $N_{\infty}(t)$, is given by

$$
N_{\infty}(t) \equiv N(\infty, t)=\int_{0}^{\infty} f(r, t) d r .
$$

For an arbitrary function $\psi(r)$ we consider its mean value $\langle\psi\rangle(t)$ in the following sense:

$$
\langle\psi\rangle(t) \equiv \frac{1}{N_{\infty}(t)} \int_{0}^{\infty} \psi(r) f(r, t) d r .
$$


We could, for example, take $\psi(r)=r$, which leads to the mean droplet radius. Alternatively, we could take $\psi(r)$ $=4 / 3 \pi r^{3}$, which leads to the mean droplet volume when spherical droplets are considered. Hence, the total volume occupied by droplets per unit volume is

$$
\vartheta(t)=N_{\infty}(t)\left\langle\frac{4}{3} \pi r^{3}\right\rangle(t) .
$$

An alternative way to derive an expression for $\vartheta(t)$ is to consider the production rate of droplets per unit volume, $J(t)$, and the time history of droplets, $\bar{r}\left(t ; t^{\prime}\right)$, which describes, for a droplet produced at production time $t^{\prime}$, the actual radius of that droplet at time $t$. With these two functions it follows that the contribution $d \vartheta(t)$ due to droplet production in the time interval $d t^{\prime}$ is

$$
d \vartheta(t)=\frac{4}{3} \pi \bar{r}^{3}\left(t ; t^{\prime}\right) J\left(t^{\prime}\right) d t^{\prime} .
$$

Hence, assuming that there are no droplets at $t=0$ and that there is no coagulation, the total volume occupied by droplets per unit volume at time $t$ is

$$
\vartheta(t)=\int_{0}^{t} \frac{4}{3} \pi \bar{r}^{3}\left(t ; t^{\prime}\right) J\left(t^{\prime}\right) d t^{\prime} .
$$

Equations (4) and (6) form two alternative expressions for the function $\vartheta(t)$. The first expression, Eq. (4), is a definition, while the second expression, Eq. (6), has been derived by physical reasoning. As stated in the Introduction, it is our objective to prove that both expressions are equivalent and, moreover, consistent with the so-called general dynamic equation.

\section{NONCONVECTED VAPOR}

We start by considering a nonconvected vapor, i.e., a vapor at rest. From mass conservation considerations it immediately follows that the mass density in this case must be constant with time:

$$
\frac{\partial \rho}{\partial t}=0
$$

\section{A. General dynamic equation}

The distribution function satisfies the so-called general dynamic equation, ${ }^{8,9}$ which, in the absence of coagulation, can be written as

$$
\frac{\partial f}{\partial t}+\frac{\partial}{\partial r}(\dot{r} f)=\delta\left(r-r^{*}\right) J .
$$

Here $\dot{r}(r, t)$ is the growth rate (the radius increase per unit time) of a droplet, $J(t) \equiv \rho \hat{J}(t)$, with $\hat{J}(t)$ the specific nucleation rate (the number of nuclei produced per unit mass, per unit time), and $\rho$ is the mass density. Furthermore, $r^{*}(t)$ is the critical radius, i.e., the radius of nuclei produced at production time $t$, and $\delta$ is the Dirac delta function. Physically, Eq. (8) expresses the balance between change in time of the distribution function at the one hand and growth and nucleation at the other hand.
At this stage we consider a number of alternative forms of the general dynamic equation to prepare an analytical solution of it. Since the mass density is constant, the division of Eq. (8) by $\rho$ leads to

$$
\frac{\partial \hat{f}}{\partial t}+\frac{\partial}{\partial r}(\dot{r} \hat{f})=\delta\left(r-r^{*}\right) \hat{J},
$$

where $\hat{f} \equiv f / \rho$. Furthermore, we integrate Eq. (9) over $r$ :

$$
\frac{\partial \hat{N}}{\partial t}+\left.(\dot{r} \hat{f})\right|_{0} ^{r}=H\left(r-r^{*}\right) \hat{J},
$$

where $\hat{N} \equiv n / \rho$ and $H$ is the Heaviside step function. Upon assuming that

$$
f(0, t)=0, \quad \forall t,
$$

and recognizing from Eq. (2) that $\hat{f} \equiv \partial \hat{N} / \partial r$ in the distributional sense, we obtain from Eq. (10) an equation for $\hat{N}(r, t)$ :

$$
\frac{\partial \hat{N}}{\partial t}+\dot{r} \frac{\partial \hat{N}}{\partial r}=H\left(r-r^{*}\right) \hat{J} .
$$

This equation expresses the balance between the time change of the number of droplets per unit mass with radii smaller than $r$ on the one hand and growth and nucleation on the other hand.

We now introduce a phase path $\bar{r}\left(t ; t_{0}\right), t \geqslant t_{0}$, as the solution of the following boundary value problem:

$$
\frac{d \bar{r}}{d t}=\dot{r}(\bar{r}, t), \quad \bar{r}\left(t_{0} ; t_{0}\right)=r^{*}\left(t_{0}\right) .
$$

Hence, $\bar{r}\left(t ; t_{0}\right)$ describes the time history of the radius of a droplet that is produced at production time $t_{0}$ and, in the mathematical sense, $\bar{r}\left(t ; t_{0}\right)$ represents a characteristic curve. While noting that for $(r, t)=(\bar{r}, t)$ :

$$
\frac{\partial \hat{N}}{\partial t}+\dot{r}(\bar{r}, t) \frac{\partial \hat{N}}{\partial r}=\frac{d}{d t} \hat{N}(\bar{r}, t),
$$

we apply Eq. (12) along the phase path $\bar{r}\left(t ; t_{0}\right)$, which leads to

$$
\frac{d}{d t} \hat{N}(\bar{r}, t)=H\left(\bar{r}-r^{*}\right) \hat{J}
$$

This equation expresses that the time increase of the number of droplets per unit mass with radii smaller than $\bar{r}$ is equal to the nucleation rate per unit mass when $\bar{r}>r^{*}$ or zero when $\bar{r}<r^{*}$. It is noted that, although growth is not present in Eq. (15) explicitly, it is included implicitly through the definition of the phase path $\bar{r}\left(t ; t_{0}\right)$. At this stage we are able to analytically construct the solution of the general dynamic equation, i.e., to determine the function $f(r, t)$.

\section{B. Analytical solution of the general dynamic equation}

Upon integration of the general dynamic equation in the form of Eq. (15) and assuming that the initial condition is

$$
\hat{N}(r, 0)=\hat{N}_{0}(r)
$$

with $\hat{N}_{0}$ a given function, we obtain 


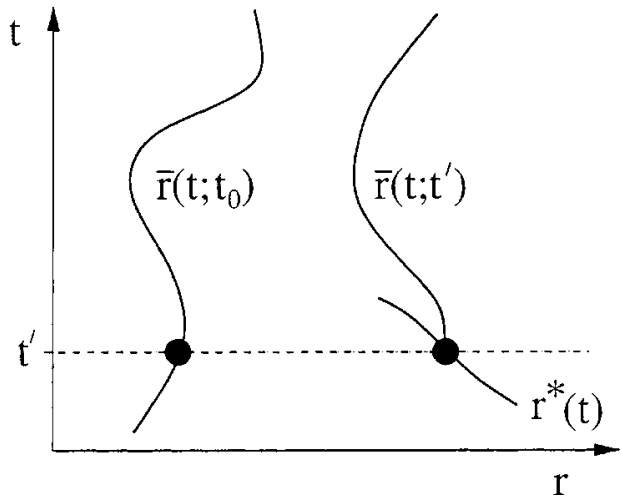

FIG. 1. Two nonintersecting phase paths in the $(r, t)$ plane for the nonconvected vapor case.

$$
\begin{aligned}
\hat{N}\left(\bar{r}\left(t ; t_{0}\right), t\right)= & \hat{N}_{0}\left(\bar{r}\left(0 ; t_{0}\right)\right)+\int_{0}^{t} H\left(\bar{r}\left(t^{\prime} ; t_{0}\right)-r^{*}\left(t^{\prime}\right)\right) \\
& \times \hat{J}\left(t^{\prime}\right) d t^{\prime} .
\end{aligned}
$$

The objective is to construct an explicit expression for the function $\hat{N}(r, t)$ in terms of $r$ and $t$. To meet this objective we will advance the argument of the Heaviside step function in the integrand of Eq. (17) in time from $t^{\prime}$ to $t$. We start by introducing a second phase path $\bar{r}\left(t ; t^{\prime}\right)$ while noting that

$$
\bar{r}\left(t^{\prime} ; t^{\prime}\right)=r^{*}\left(t^{\prime}\right),
$$

by definition, viz. Eq. (13). Hence, two phase paths are involved, which are both shown schematically in Fig. 1. We will now show that two nonidentical phase paths cannot intersect by a contradiction argument.

Suppose that two nonidentical phase paths do intersect, then both paths are solutions of a boundary value problem similar to Eq. (13) but with the boundary condition replaced by the intersection point in terms of a radius and a time instant. Since the function $\dot{r}(r, t)$ is considered single-valued, however, the boundary value problem has a unique solution and the two phase paths must be identical for all time instants larger than in the intersection instant. Similar reasoning leads to the conclusion that the two phase paths must also be identical for all time instants smaller than in the intersection instant. Hence, we have reached a contradiction and have indeed proved that two nonidentical phase paths cannot intersect.

It is noted that this result can also obtained in a more general way by employing the integral formulation of Liouville's equation, ${ }^{10}$ which expresses the conservation of probability. Using the Liouville equation, one can show that if the starting points of the two phase paths are not identical then the probability to have an intersection point is identically zero.

As a result of this observation, we deduce that if $\bar{r}\left(t^{\prime} ; t_{0}\right)>r^{*}\left(t^{\prime}\right) \equiv \bar{r}\left(t^{\prime} ; t^{\prime}\right)$ then $\bar{r}\left(t ; t_{0}\right)>\bar{r}\left(t ; t^{\prime}\right)$ for all $t$. A similar result holds for the $<$ sign. As a consequence we may write

$$
H\left(\bar{r}\left(t^{\prime} ; t_{0}\right)-r^{*}\left(t^{\prime}\right)\right) \equiv H\left(\bar{r}\left(t ; t_{0}\right)-\bar{r}\left(t ; t^{\prime}\right)\right) .
$$

Upon substitution of this identity into Eq. (17), we note that the resulting integral does not contain the phase path $\bar{r}\left(t^{\prime} ; t_{0}\right)$ anymore, except for the two points $\bar{r}\left(0 ; t_{0}\right)$ and $\bar{r}\left(t ; t_{0}\right)$. The first point can be written as

$$
\bar{r}\left(0 ; t_{0}\right)=r_{0}\left(\bar{r}\left(t ; t_{0}\right), t\right),
$$

where the function $r_{0}(r, t)$ represents backward time integration along the phase path $\bar{r}$ from $t=t$ to $t=0$ starting at $\bar{r}\left(t ; t_{0}\right)$. Therefore, writing $r=\bar{r}\left(t ; t_{0}\right)$, we obtain

$$
\hat{N}(r, t)=\hat{N}_{0}\left(r_{0}(r, t)\right)+\int_{0}^{t} H\left(r-\bar{r}\left(t ; t^{\prime}\right)\right) \hat{J}\left(t^{\prime}\right) d t^{\prime} .
$$

This equation has a very clear interpretation. The first term on the right hand side represents, without nucleation, the number of droplets with radii smaller than $r$ at time $t$, which is equal to the number of droplets with radii smaller than $r_{0}$ at time $t=0$, which is a direct result of the fact that droplets "cannot pass" the phase path between $\left(r_{0}, 0\right)$ and $(r, t)$. The second term on the right hand side represents the number of nucleated droplets with radii smaller than $r$ at time $t$, as a time integral of the nucleation term $J(t)$ multiplied by a switch to select the droplets that remain small enough to fall into the class $\hat{N}(r, t)$.

Hence, we indeed have obtained an explicit expression of $\hat{N}(r, t)$ in terms of $r$ and $t$. It is noted, however, that in contrast to Eq. (17) where one single phase path $\bar{r}\left(t^{\prime} ; t_{0}\right)$ is used, Eq. (21) requires a range of such phase paths $\bar{r}\left(t ; t^{\prime}\right)$, with $0 \leqslant t^{\prime} \leqslant t$. The analytical solution of the general dynamic equation in terms of the distribution function is readily obtained from Eq. (21) by differentiation with respect to $r$ and multiplication by $\rho$ :

$$
\begin{aligned}
f(r, t)= & f_{0}\left(r_{0}(r, t)\right) \frac{\partial r_{0}}{\partial r}(r, t) \\
& +\int_{0}^{t} \delta\left(r-\bar{r}\left(t ; t^{\prime}\right)\right) J\left(t^{\prime}\right) d t^{\prime},
\end{aligned}
$$

where the function $f_{0}(r)$ is defined as

$$
f_{0}(r) \equiv \frac{d N_{0}}{d r}, \quad N_{0}(r)=\rho \hat{N}_{0}(r) .
$$

When one substitutes the analytical solution, Eq. (22), into the general dynamic equation, Eq. (8), for verification purposes, it is worthwhile to note that the following compatibility condition holds:

$$
\frac{\partial}{\partial t} r_{0}(r, t)=-\dot{r}(r, t) \frac{\partial}{\partial r} r_{0}(r, t) .
$$

This condition reflects that, when the end point $(r, t)$ of the phase path starting in $\left(r_{0}(r, t), 0\right)$ is varied along the phase path, the starting point is independent of this variation, i.e.,

$$
d r=\dot{r} d t \Rightarrow d r_{0}=\frac{\partial r_{0}}{\partial t} d t+\frac{\partial r_{0}}{\partial r} d r=0 .
$$

The analytical solution in the form of Eq. (22) can be related to existing literature on the subject by distinguishing between the two terms on the right-hand side.

The first term on the right-hand side of Eq. (22), 


$$
f_{0}\left(r_{0}(r, t)\right) \frac{\partial r_{0}}{\partial r}(r, t)
$$

is a generalization of the analytic solution obtained for the case without nucleation in Refs. 11 and 12. When we adopt the assumption made in Ref. 11 that the growth rate function $\dot{r}(r, t)$ can be written as

$$
\dot{r}(r, t)=R(r) T(t),
$$

then, employing the phase path definition Eq. (13), it is easy to show that the function $r_{0}(r, t)$ is implicitly defined by

$$
\int_{0}^{r_{0}(r, t)} \frac{d r^{\prime}}{R\left(r^{\prime}\right)}=\int_{0}^{r} \frac{d r^{\prime}}{R\left(r^{\prime}\right)}-\int_{0}^{t} T\left(t^{\prime}\right) d t^{\prime},
$$

which is an explicit form of the condition mentioned in Ref. 12. The differentiation of Eq. (28) with respect to $r$ leads to

$$
\frac{\partial r_{0}}{\partial r}(r, t)=\frac{R\left(r_{0}(r, t)\right)}{R(r)},
$$

which, upon substitution into expression (26), leads to the analytic solution presented in Refs. 11 and 12.

The second term on the right-hand side of Eq. (22),

$$
\int_{0}^{t} \delta\left(r-\bar{r}\left(t ; t^{\prime}\right)\right) J\left(t^{\prime}\right) d t^{\prime}
$$

can be shown to be identical to

$$
\begin{aligned}
& \frac{1}{\mu\left(t ; t_{0}\right)} \int_{0}^{t} \mu\left(t^{\prime} ; t_{0}\right) \delta\left(\bar{r}\left(t^{\prime} ; t_{0}\right)-r^{*}\left(t^{\prime}\right)\right) J\left(t^{\prime}\right) d t^{\prime}, \\
& \bar{r}\left(t ; t_{0}\right)=r,
\end{aligned}
$$

which is the exact solution that is obtained in Refs. 13, 14, and 15 . Here $\mu\left(t ; t_{0}\right)$ is the so-called integrating factor: ${ }^{16}$

$$
\mu\left(t ; t_{0}\right) \equiv \exp \int_{0}^{t} \frac{\partial \dot{r}}{\partial r}\left(\bar{r}\left(t^{\prime} ; t_{0}\right), t^{\prime}\right) d t^{\prime} .
$$

The identity of expressions (30) and (31), however, is not trivial. First we note that

$$
\begin{aligned}
\delta\left(r-\bar{r}\left(t ; t^{\prime}\right)\right) & =\frac{\partial}{\partial r} H\left(r-\bar{r}\left(t ; t^{\prime}\right)\right) \\
& =\frac{\partial}{\partial r} H\left(\bar{r}\left(t^{\prime} ; t_{0}\right)-r^{*}\left(t^{\prime}\right)\right) \\
& =\frac{\partial}{\partial r} \int_{0}^{\bar{r}\left(t^{\prime} ; t_{0}\right)} \delta\left(\bar{r}-r^{*}\left(t^{\prime}\right)\right) d \tilde{r} \\
& =\delta\left(\bar{r}\left(t^{\prime} ; t_{0}\right)-r^{*}\left(t^{\prime}\right)\right) \frac{\partial \bar{r}\left(t^{\prime} ; t_{0}\right)}{\partial r},
\end{aligned}
$$

where it is understood that $t_{0}$ is determined by $r=\bar{r}\left(t ; t_{0}\right)$.

Second, we employ a differential equation that is related to the Reynolds' transport theorem of fluid mechanics. The differential equation indicated expresses the time derivative of the Jacobian of the transformation from material coordinates (initial coordinates of fluid particles) to spatial coordinates (actual coordinates of fluid particles) as the product of the divergence of the velocity field and the Jacobian. ${ }^{17}$ In our case, $r$ acts as the material coordinate, $\bar{r}$ acts as the spatial coordinate, $\dot{r}$ acts as the velocity field, and $\partial \dot{r} / \partial r$ acts as the divergence of the velocity field. As a result, the differential equation indicated becomes

$$
\frac{\partial}{\partial t^{\prime}}\left(\frac{\partial \bar{r}\left(t^{\prime} ; t_{0}\right)}{\partial r}\right)=\frac{\partial \dot{r}}{\partial r}\left(\bar{r}\left(t^{\prime} ; t_{0}\right), t^{\prime}\right) \frac{\partial \bar{r}\left(t^{\prime} ; t_{0}\right)}{\partial r} .
$$

The solution of this differential equation gives

$$
\frac{\partial \bar{r}\left(t^{\prime} ; t_{0}\right)}{\partial r}=\frac{\mu\left(t^{\prime} ; t_{0}\right)}{\mu\left(t ; t_{0}\right)} .
$$

The substitution of Eq. (33) and Eq. (35) into expression (30) shows the identity of expressions (30) and (31).

Finally, it is noted that the present solution route via a solution of the preintegrated form of the general dynamic equation, viz. Eq. (12), directly leads to expression (30), which is explicit in terms of $r$. This is a necessary condition since we need to integrate $f(r, t)$ with respect to $r$ in Sec. III C. In contrast, the conventional solution route via solution of the original general dynamic equation, Eq. (8), leads to expression (31), which is not explicit in terms of $r$.

\section{Equivalence result for nonconvected vapor}

Our objective is to represent the mean value of a function $\psi(r)$ defined by Eq. (3) by an equivalent integral over $t$ under the condition that the vapor is initially free of droplets, i.e., $N_{0}=0$ (and hence $f_{0}=0$ ). Substitution of the analytical solution, Eq. (22), for $f(r, t)$ into Eq. (3) leads to

$$
N_{\infty}(t)\langle\psi\rangle(t)=\int_{0}^{\infty} \psi(r) \int_{0}^{t} \delta\left(r-\bar{r}\left(t ; t^{\prime}\right)\right) J\left(t^{\prime}\right) d t^{\prime} d r .
$$

The function $\psi(r)$ can be put under the time integral, and subsequently the order of integration can be exchanged. Finally, by employing the delta function property, we obtain

$$
\langle\psi\rangle(t)=\frac{1}{N_{\infty}(t)} \int_{0}^{t} \psi\left(\bar{r}\left(t ; t^{\prime}\right)\right) J\left(t^{\prime}\right) d t^{\prime} .
$$

Hence, the equivalence of the radius integral formulation, Eq. (3), and the time integral formulation, Eq. (37), has been proved:

$$
\int_{0}^{\infty} \psi(r) f(r, t) d r=\int_{0}^{t} \psi\left(\bar{r}\left(t ; t^{\prime}\right)\right) J\left(t^{\prime}\right) d t^{\prime} .
$$

\section{Specific choices for $\psi(r)$}

\section{Unity: $\psi(r)=1$}

With this choice, Eq. (38) imediately leads to

$$
N_{\infty}(t)=\int_{0}^{t} J\left(t^{\prime}\right) d t^{\prime}
$$

which expresses that the number of droplets per unit volume is equal to the integral over time of the nucleation rate. 


\section{Dirac delta function: $\psi(r)=\delta\left(r_{0}-r\right)$}

With this choice, Eq. (38) leads to

$$
f\left(r_{0}, t\right)=\int_{0}^{t} \delta\left(r_{0}-\bar{r}\left(t ; t^{\prime}\right)\right) J\left(t^{\prime}\right) d t^{\prime},
$$

which is just Eq. (22).

\section{Heaviside step function: $\psi(r)=H\left(r_{0}-r\right)$}

With this choice, Eq. (38) leads to

$$
N\left(r_{0}, t\right)=\int_{0}^{t} H\left(r_{0}-\bar{r}\left(t ; t^{\prime}\right)\right) J\left(t^{\prime}\right) d t^{\prime},
$$

which is just Eq. (21) multiplied by $\rho$.

\section{4. kth moment: $\psi(r)=r^{k}$}

With this choice, Eq. (38) leads to

$$
N_{\infty}(t)\left\langle r^{k}\right\rangle(t)=\int_{0}^{\infty} r^{k} f(r, t) d r=\int_{0}^{t} \bar{r}^{k}\left(t ; t^{\prime}\right) J\left(t^{\prime}\right) d t^{\prime} .
$$

The quantity $N_{\infty}(t)\left\langle r^{k}\right\rangle(t)$ is commonly referred to as the " $k$ th moment of the distribution function." The establishment of Eq. (42) is, in fact, the motivation for the present paper; the radius integral is used within investigations with respect to aerosol dynamics, while the time integral is used within investigations with respect to nonequilibrium condensation in transonic nozzle flows. The specific choice $k=3$ directly leads to the equivalence of expressions (4) and (6).

\section{E. Time derivative of $\boldsymbol{N}_{\infty}(t)\langle\psi\rangle(t)$}

The derivative of $N_{\infty}(t)\langle\psi\rangle(t)$ with respect to $t$, with $\psi(r)=r^{k}$, is frequently used within the so-called "method of moments" to establish a closed set of ordinary differential equations to be solved in time for the moments $\left\langle r^{k}\right\rangle(t)$. We will derive the time derivative of $N_{\infty}(t)\langle\psi\rangle(t)$, with $\psi(r)$ arbitrary, and show how both the radius integral formulation and the time integral formulation lead to the same result, as must be expected.

We start by differentiating the radius integral Eq. (3):

$$
\frac{d}{d t} N_{\infty}(t)\langle\psi\rangle(t)=\int_{0}^{\infty} \psi(r) \frac{\partial f}{\partial t} d r .
$$

By employing the general dynamic equation, Eq. (8), the integral can be written as

$$
\int_{0}^{\infty} \psi(r)\left\{\delta\left(r-r^{*}\right) J-\frac{\partial}{\partial r}(\dot{r} f)\right\} d r,
$$

which, upon partial integration and using

$$
f(0, t)=f(\infty, t)=0,
$$

leads to

$$
\frac{d}{d t} N_{\infty}(t)\langle\psi\rangle(t)=\psi\left(r^{*}\right) J+N_{\infty}(t)\left\langle\dot{r} \frac{d \psi}{d r}\right\rangle(t) .
$$

$$
\frac{d}{d t} N_{\infty}(t)\langle\psi\rangle(t)=\psi(\bar{r}(t ; t)) J+\int_{0}^{t} \frac{d \psi}{d r}(\bar{r}) \frac{d \bar{r}}{d t} J d t^{\prime},
$$

where $\bar{r}$ in the integrand represents $\bar{r}\left(t ; t^{\prime}\right)$.

Since $d \bar{r} / d t=\dot{r}(\bar{r}, t)$, viz. Eq. (13), and

$$
\int_{0}^{t} \frac{d \psi}{d r}(\bar{r}) \dot{r}(\bar{r}, t) J d t^{\prime}=N_{\infty}(t)\left\langle\frac{d \psi}{d r} \dot{r}\right\rangle(t),
$$

which follows from the equivalence result, viz. Eq. (38), we again obtain Eq. (46) from Eq. (47). This concludes the developments for the nonconvected vapor case.

\section{CONVECTED VAPOR}

We will now extend the obtained results to the case of convected vapor. In contrast to the case of nonconvected vapor, the mass density is not a constant. Instead, we have to satisfy the mass conservation law for $\rho(\mathbf{x}, t)$ :

$$
\frac{\partial \rho}{\partial t}+\frac{\partial}{\partial x_{j}}\left(\rho u_{j}\right)=0,
$$

where we use the summation convention and where $\mathbf{u}(\mathbf{x}, t)$ represents the velocity field.

\section{A. General dynamic equation}

We start by extending the general dynamic equation, Eq. (8), to account for convection. The distribution function in this case is not only function of radius and time, but also a function of the spatial coordinate $\mathbf{x}$.

Furthermore $f(r, \mathbf{x}, t)$ is defined such that for any given $r>0, \mathbf{x}$ and $t>0$, the number of droplets per unit volume with radii smaller than $r$ is given by

$$
N(r, \mathbf{x}, t)=\int_{0}^{r} f\left(r^{\prime}, \mathbf{x}, t\right) d r^{\prime} .
$$

The general dynamic equation, in the absence of coagulation, can be written as ${ }^{8,9}$

$$
\frac{\partial f}{\partial t}+\frac{\partial}{\partial r}(\dot{r} f)+\frac{\partial}{\partial x_{j}}\left(u_{j} f\right)=\delta\left(r-r^{*}\right) J .
$$

Here both the growth rate $\dot{r}(r, \mathbf{x}, t)$, the nucleation rate $J(\mathbf{x}, t)$, and the critical radius $r^{*}(\mathbf{x}, t)$ are functions of the spatial coordinate as well. The nucleation rate is defined as $J(\mathbf{x}, t) \equiv \rho(\mathbf{x}, t) \hat{J}(\mathbf{x}, t)$, where $\hat{J}(\mathbf{x}, t)$ is the specific nucleation rate. Physically, Eq. (51) expresses the balance between the change in time of the distribution function on the one hand and growth, convection, and nucleation on the other hand.

Similar to the situation of a nonconvected vapor we consider a number of alternative forms of the general dynamic equation to prepare an analytical solution of it. Writing $f$ $\equiv \rho \hat{f}$ in Eq. (51) and then using Eq. (49) leads to

$$
\frac{\partial \hat{f}}{\partial t}+\frac{\partial}{\partial r}(\dot{r} \hat{f})+u_{j} \frac{\partial \hat{f}}{\partial x_{j}}=\delta\left(r-r^{*}\right) \hat{J} .
$$

We integrate Eq. (52) over $r$ :

$$
\frac{\partial \hat{N}}{\partial t}+\left.(\dot{r} \hat{f})\right|_{0} ^{r}+u_{j} \frac{\partial \hat{N}}{\partial x_{j}}=H\left(r-r^{*}\right) \hat{J},
$$




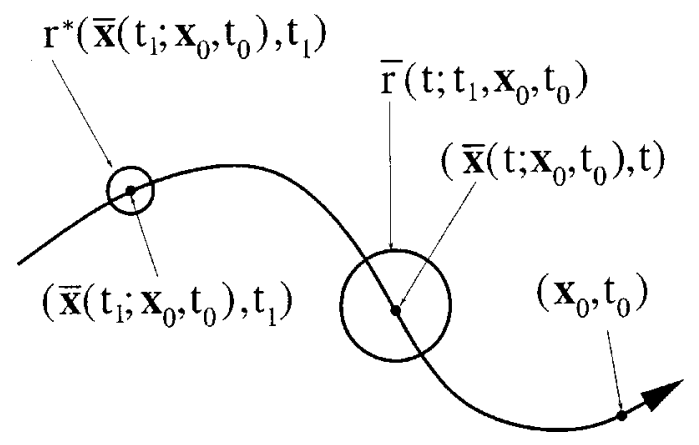

FIG. 2. An illustration of the phase path definition for the convected vapor case.

where again $\hat{N} \equiv N / \rho$. Upon assuming that

$$
f(0, \mathbf{x}, t)=0, \quad \forall \mathbf{x}, t,
$$

and recognizing from Eq. (50) that $\hat{f} \equiv \partial \hat{N} / \partial r$ in the distributional sense, we obtain from Eq. (53) an equation for $\hat{N}(r, \mathbf{x}, t)$ :

$$
\frac{\partial \hat{N}}{\partial t}+\dot{r} \frac{\partial \hat{N}}{\partial r}+u_{j} \frac{\partial \hat{N}}{\partial x_{j}}=H\left(r-r^{*}\right) \hat{J} .
$$

This equation expresses the balance between the time change of the number of droplets per unit mass with radii smaller than $r$ on the one hand and growth, convection, and nucleation on the other hand.

Up to this stage, the developments are fully similar to the case of nonconvected vapor. In defining a phase path, however, things become slightly more complex, in the sense that it consists of four variables instead of one. In addition, we prefer to use a slightly more general definition of the phase path in view of developments described below. We define a phase path in terms of a set of four functions: the radius coordinate $\bar{r}\left(t ; t_{1}, \mathbf{x}_{0}, t_{0}\right)$ and the spatial coordinates $\overline{\mathbf{x}}\left(t ; \mathbf{x}_{0}, t_{0}\right)$, defined as the solution of the following two coupled boundary value problems:

$$
\frac{d \overline{\mathbf{x}}}{d t}=\mathbf{u}(\overline{\mathbf{x}}, t), \quad \overline{\mathbf{x}}\left(t_{0} ; \mathbf{x}_{0}, t_{0}\right)=\mathbf{x}_{0},
$$

and

$$
\frac{d \bar{r}}{d t}=\dot{r}(\bar{r}, \overline{\mathbf{x}}, t), \quad \bar{r}\left(t_{1} ; t_{1}, \mathbf{x}_{0}, t_{0}\right)=r^{*}\left(\overline{\mathbf{x}}\left(t_{1} ; \mathbf{x}_{0}, t_{0}\right), t_{1}\right) .
$$

Hence, $\bar{r}$ describes the time evolution of the radius of a droplet produced at $t=t_{1}$, while $\overline{\mathbf{x}}$ describes the time evolution of the position of that droplet including the point $\left(\mathbf{x}_{0}, t_{0}\right)$; see the illustration in Fig. 2. It is noted that the function $\overline{\mathbf{x}}$ is defined not only for $t \geqslant t_{0}$ but also for $t<t_{0}$, which is convenient in a later stage.

While noting that for $(r, \mathbf{x}, t)=(\bar{r}, \overline{\mathbf{x}}, t)$ :

$$
\frac{\partial \hat{N}}{\partial t}+\dot{r} \frac{\partial \hat{N}}{\partial r}+u_{j} \frac{\partial \hat{N}}{\partial x_{j}}=\frac{d}{d t} \hat{N}(\bar{r}, \overline{\mathbf{x}}, t),
$$

we apply Eq. (55) along the phase path $(\bar{r}, \overline{\mathbf{x}})$, which leads to

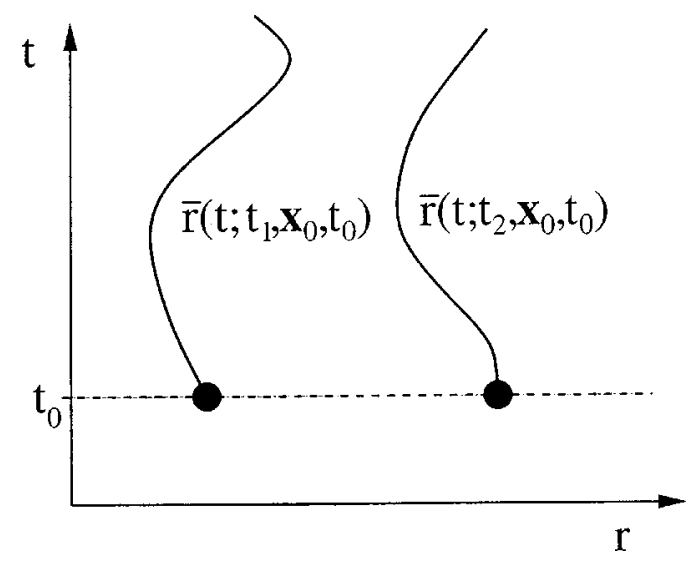

FIG. 3. Two phase paths projected onto the $(r, t)$ plane for the convected vapor case.

$$
\frac{d}{d t} \hat{N}(\bar{r}, \overline{\mathbf{x}}, t)=H\left(\bar{r}-r^{*}(\overline{\mathbf{x}}, t)\right) \hat{J}(\overline{\mathbf{x}}, t) .
$$

This equation expresses that the time increase of the number of droplets per unit mass with radii smaller than $\bar{r}$ is equal to the nucleation rate per unit mass when $\bar{r}>r^{*}$ or zero when $\bar{r}<r^{*}$. At this stage we are able to analytically construct the solution of the general dynamic equation, i.e., to determine the function $f(r, \mathbf{x}, t)$.

\section{B. Analytical solution of the general dynamic equation}

Upon time integration of the general dynamic equation in the form of Eq. (59) while assuming that

$$
\hat{N}(r, \mathbf{x}, 0)=0, \quad \forall r, \mathbf{x},
$$

we obtain

$$
\begin{aligned}
\hat{N}(\bar{r}(t), \overline{\mathbf{x}}(t), t)= & \int_{0}^{t} H\left(\bar{r}\left(t^{\prime}\right)-r^{*}\left(\overline{\mathbf{x}}\left(t^{\prime}\right), t^{\prime}\right)\right) \\
& \times \hat{J}\left(\overline{\mathbf{x}}\left(t^{\prime}\right), t^{\prime}\right) d t^{\prime},
\end{aligned}
$$

where we have abreviated $\bar{r}\left(t ; t_{1}, \mathbf{x}_{0}, t_{0}\right)$ as $\bar{r}(t)$ and $\overline{\mathbf{x}}\left(t ; \mathbf{x}_{0}, t_{0}\right)$ as $\overline{\mathbf{x}}(t)$. Similar to the nonconvected vapor case we want to construct an explicit expression for the function $\hat{N}(r, \mathbf{x}, t)$ in terms of $r, \mathbf{x}$ and $t$.

To this end, we consider a set of two phase paths defined as $\left(\bar{r}\left(t ; t_{1}, \mathbf{x}_{0}, t_{0}\right), \overline{\mathbf{x}}\left(t ; \mathbf{x}_{0}, t_{0}\right)\right)$ and $\left(\bar{r}\left(t ; t_{2}, \mathbf{x}_{0}, t_{0}\right), \overline{\mathbf{x}}\left(t ; \mathbf{x}_{0}, t_{0}\right)\right)$, of which projections onto the $(r, t)$ plane and the $\mathbf{x}$ space are shown schematically in Fig. 3 and Fig. 4, respectively. A similar reasoning as is presented for the nonconvected vapor case leads to the conclusion that the two phase paths considered cannot intersect. Since the projections of the two phase paths onto the $\mathbf{x}$ space are coinciding, see Fig. 4, this means that $\bar{r}\left(t ; t_{1}, \mathbf{x}_{0}, t_{0}\right) \neq \bar{r}\left(t ; t_{2}, \mathbf{x}_{0}, t_{0}\right)$ for all $t$.

With this information we inspect the argument of the Heaviside step function in Eq. (61) and advance it in time from $t^{\prime}$ to $t$, knowing that the sign of it is invariant under this operation. First, we advance $\bar{r}\left(t^{\prime}\right)$, or equivalently $\bar{r}\left(t^{\prime} ; t_{1}, \mathbf{x}_{0}, t_{0}\right)$, in time to $\bar{r}\left(t ; t_{1}, \mathbf{x}_{0}, t_{0}\right)$. As a consequence, only this single point of the phase path $\bar{r}\left(t^{\prime}\right)$ occurs in Eq. 


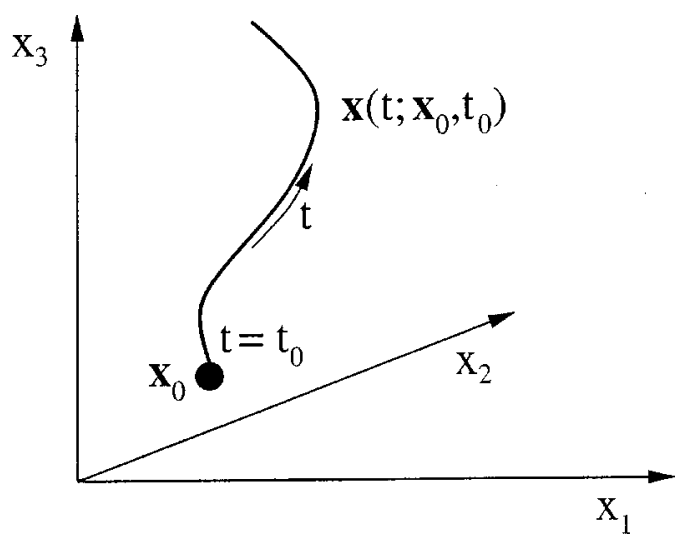

FIG. 4. Two phase paths projected onto the $\mathbf{x}$ space for the convected vapor case, showing coinciding projections.

(61), and we may simply write $r=\bar{r}\left(t ; t_{1}, \mathbf{x}_{0}, t_{0}\right)$ instead. Second, we advance $r^{*}\left(\mathbf{x}\left(t^{\prime}\right), t^{\prime}\right)$ in time to $\bar{r}\left(t ; t^{\prime}, \mathbf{x}_{0}, t_{0}\right)$, which we write as $\bar{r}\left(t ; t^{\prime}\right)$ for simplicity. Furthermore, we may choose $t_{0}=t$ and $\mathbf{x}_{0}=\mathbf{x}$, yielding $\overline{\mathbf{x}}(t)=\mathbf{x}$. The substitution of these expressions into Eq. (61) then leads to

$$
\hat{N}(r, \mathbf{x}, t)=\int_{0}^{t} H\left(r-\bar{r}\left(t ; t^{\prime}\right)\right) \hat{J}\left(\overline{\mathbf{x}}\left(t^{\prime}\right), t^{\prime}\right) d t^{\prime},
$$

with

$$
\begin{aligned}
& \overline{\mathbf{x}}\left(t^{\prime}\right):=\overline{\mathbf{x}}\left(t^{\prime} ; \mathbf{x}, t\right), \\
& \bar{r}\left(t ; t^{\prime}\right):=\bar{r}\left(t ; t^{\prime}, \mathbf{x}, t\right) .
\end{aligned}
$$

Differentiation of this equation with respect to $r$ and multiplication with $\rho(\mathbf{x}, t)$ finally gives the explicit expression for the distribution function:

$$
f(r, \mathbf{x}, t)=\rho(\mathbf{x}, t) \int_{0}^{t} \delta\left(r-\bar{r}\left(t ; t^{\prime}\right)\right) \hat{J}\left(\overline{\mathbf{x}}\left(t^{\prime}\right), t^{\prime}\right) d t^{\prime} .
$$

This expression is very similar to the one obtained for nonconvected vapor, Eq. (22), although there are two differences. First the time integral represents traveling with the vapor and, second, the dependency of the mass density along the phase paths is included.

\section{Equivalence result for convected vapor}

For convected vapor the mean value of a function $\psi(r)$ is defined as

$$
\langle\psi\rangle(\mathbf{x}, t) \equiv \frac{1}{N_{\infty}(\mathbf{x}, t)} \int_{0}^{\infty} \psi(r) f(r, \mathbf{x}, t) d r,
$$

where the number density $N_{\infty}(\mathbf{x}, t)$ is defined as

$$
N_{\infty}(\mathbf{x}, t) \equiv N(\infty, \mathbf{x}, t) .
$$

The substitution of Eq. (64) as the analytical solution of $f(r, \mathbf{x}, t)$ into Eq. (65) and the reversed order of integration leads to

$$
\langle\psi\rangle(\mathbf{x}, t)=\frac{\rho(\mathbf{x}, t)}{N_{\infty}(\mathbf{x}, t)} \int_{0}^{t} \psi\left(\bar{r}\left(t ; t^{\prime}\right)\right) \hat{J}\left(\overline{\mathbf{x}}\left(t^{\prime}\right), t^{\prime}\right) d t^{\prime} .
$$

A comparison of Eq. (65) and Eq. (67) yields the equivalence result:

$$
\int_{0}^{\infty} \psi(r) f(r, \mathbf{x}, t) d r=\rho(\mathbf{x}, t) \int_{0}^{t} \psi\left(\bar{r}\left(t ; t^{\prime}\right)\right) \hat{J}\left(\overline{\mathbf{x}}\left(t^{\prime}\right), t^{\prime}\right) d t^{\prime},
$$

which can be written more compactly:

$$
\int_{0}^{\infty} \psi(r) \hat{f}(r, \mathbf{x}, t) d r=\int_{0}^{t} \psi\left(\bar{r}\left(t ; t^{\prime}\right)\right) \hat{J}\left(\overline{\mathbf{x}}\left(t^{\prime}\right), t^{\prime}\right) d t^{\prime} .
$$

\section{Specific choices for $\psi(r)$}

\section{Unity: $\psi(r)=1$}

With this choice, Eq. (69) imediately leads to

$$
\hat{N}_{\infty}(\mathbf{x}, t)=\int_{0}^{t} \hat{J}\left(\mathbf{x}\left(t^{\prime}\right), t^{\prime}\right) d t^{\prime},
$$

which expresses that the number of droplets per unit mass is equal to the integral over time of the specific nucleation rate while traveling with the vapor.

2. Dirac delta function: $\psi(r)=\delta\left(r_{0}-r\right)$

With this choice, Eq. (69) leads to

$$
\hat{f}\left(r_{0}, \mathbf{x}, t\right)=\int_{0}^{t} \delta\left(r_{0}-\bar{r}\left(t ; t^{\prime}\right)\right) \hat{J}\left(\overline{\mathbf{x}}\left(t^{\prime}\right), t^{\prime}\right) d t^{\prime},
$$

which is just Eq. (64).

\section{Heaviside step function: $\psi(r)=H\left(r_{0}-r\right)$}

With this choice, Eq. (69) leads to

$$
\hat{N}\left(r_{0}, \mathbf{x}, t\right)=\int_{0}^{t} H\left(r_{0}-\bar{r}\left(t ; t^{\prime}\right)\right) \hat{J}\left(\mathbf{x}\left(t^{\prime}\right), t^{\prime}\right) d t^{\prime},
$$

which is just Eq. (62).

\section{4. kth moment: $\psi(r)=r^{k}$}

With this choice, Eq. (69) leads to

$$
\int_{0}^{\infty} r^{k} \hat{f}(r, \mathbf{x}, t) d r=\int_{0}^{t} \bar{r}^{k}\left(t ; t^{\prime}\right) \hat{J}\left(\overline{\mathbf{x}}\left(t^{\prime}\right), t^{\prime}\right) d t^{\prime} .
$$

\section{E. Material time derivative of $N_{\infty}(\mathrm{x}, t)\langle\psi\rangle(\mathrm{x}, t)$}

Finally, we develop an expression for the so-called material time derivative of $N_{\infty}(\mathbf{x}, t)\langle\psi\rangle(\mathbf{x}, t)$. The material time derivative is defined by means of the following differential operator:

$$
\frac{D}{D t} \equiv \frac{\partial}{\partial t}+u_{j} \frac{\partial}{\partial x_{j}} .
$$

We evaluate

$$
\frac{D}{D t} N_{\infty}(\mathbf{x}, t)\langle\psi\rangle(\mathbf{x}, t)=\int_{0}^{\infty} \psi(r) \frac{D}{D t} f(r, \mathbf{x}, t) d r .
$$

By employing the general dynamic equation, Eq. (51), the integral can be written as 


$$
\int_{0}^{\infty} \psi(r)\left\{\delta\left(r-r^{*}\right) J-\frac{\partial}{\partial r}(\dot{r} f)\right\} d r
$$

which, upon partial differentiation and using

$$
f(0, \mathbf{x}, t)=f(\infty, \mathbf{x}, t)=0,
$$

leads to

$$
\frac{D}{D t} N_{\infty}(\mathbf{x}, t)\langle\psi\rangle(\mathbf{x}, t)=\psi\left(r^{*}\right) J+N_{\infty}(\mathbf{x}, t)\left\langle\dot{r} \frac{d \psi}{d r}\right\rangle(\mathbf{x}, t),
$$

which is completely similar to Eq. (46).

\section{CONCLUSIONS AND DISCUSSION}

It has been proved that two different integral representations of droplet distribution moments reported in the literature are equivalent and, moreover, consistent with the general dynamic equation.

The underlying key element of the proof consists of analytical solution of the general dynamic equation in the form of an explicit expression of the droplet size distribution function in terms of an integral over time, involving phase paths (growth and convection) on the one hand and droplet production (nucleation) on the other hand.

The obtained analytical solution has the following properties.

(1) It includes a generalization of an existing analytical solution for the case without nucleation.

(2) It is in agreement with the existing analytical solution for the case with nucleation.

(3) It is derived from the preintegrated general dynamic equation instead of the general dynamic equation itself.

(4) It is explicit in terms of the droplet radius, in contrast to existing analytical solutions.

Both the nonconvected vapor case and the convected vapor case have been presented, leading to detailed expressions of the time integral representation of the distribution moments.

It is noted that droplet removal due to evaporation is not included in the present investigation. It is expected, however, that the integral equivalence still holds for the case that drop- let removal is included and that a proof can be obtained, employing a procedure similar to the one employed in the present investigation.

\section{ACKNOWLEDGMENTS}

The author wishes to express his gratitude to Professor M. E. H. van Dongen of the Eindhoven University of Technology and Professor E. W. C. van Groesen of the University of Twente, for sharing their ideas on the subject.

\footnotetext{
${ }^{1} \mathrm{~K}$. Oswatitsch, "Kondensationserscheinungen in überschalldüsen," Z. Angew. Math. Mech. 22, 1 (1942).

${ }^{2}$ P. G. Hill, "Condensation of water vapour during supersonic expansion in nozzles," J. Fluid Mech. 25, 593 (1966).

${ }^{3}$ P. A. Blythe and C. J. Shih, "Condensation shocks in nozzle flows," J. Fluid Mech. 76, 593 (1976).

${ }^{4}$ J. H. Clarke and C. F. Delale, "Nozzle flows with nonequilibrium condensation," Phys. Fluids 29, 1398 (1986).

${ }^{5}$ C. F. Delale, M. J. E. H. Muitjens, and M. E. H. van Dongen, “Asymptotic solution and numerical simulation of homogeneous condensation in expansion cloud chambers," J. Chem. Phys. 105, 8804 (1996).

${ }^{6} \mathrm{~S}$. Adam and G. Schnerr, "Instabilities and bifurcation of non-equilibrium two-phase flows," J. Fluid Mech. 348, 1 (1997).

${ }^{7}$ D. L. Wright, S. Yu, P. S. Kasibhatla, R. McGraw, S. E. Schwartz, V. K. Saxena, and G. K. Yue, "Retrieval of aerosol properties from moments of the particle size distribution for kernels involving the step function: Cloud droplet activation," J. Aerosol Sci. 33, 319 (2002).

${ }^{8}$ J. H. Seinfeld, Atmospheric Chemistry and Physics of Air Pollution (Wiley, New York, 1986).

${ }^{9}$ M. M. R. Williams and S. K. Loyalka, Aerosol Science Theory and Practice (Pergamon, Oxford, 1991).

${ }^{10} \mathrm{C}$. Cercignani, The Boltzmann Equation and its Applications (SpringerVerlag, New York, 1988).

${ }^{11}$ J. R. Brock, "New aspects of aerosol growth processes," Aerosol Sci. Technol. 2, 109 (1983).

${ }^{12}$ S. K. Loyalka and J. W. Park, "Aerosol growth by condensation: A generalization of Mason's formula," J. Colloid Interface Sci. 125, 712 (1988).

${ }^{13}$ F. Gelbard and J. H. Seinfeld, "Exact solution of the general dynamic equation for aerosol growth by condensation," J. Colloid Interface Sci. 68, 173 (1979).

${ }^{14}$ D. Katoshevski and J. H. Seinfeld, "Analytical solution of the multicomponent aerosol general dynamic equation-without coagulation," Aerosol. Sci. Technol. 27, 541 (1997).

${ }^{15}$ J. M. Fernández-Díaz, M. A. Rodríguez Braña, B. Arganza García, C. González-Pola Muñiz, and P. J. García Nieto, "Analytical solution of the aerosol rigorous general dynamic equation without coagulation in multidimension," Aerosol Sci. Technol. 31, 3 (1999).

${ }^{16}$ W. E. Boyce and R. C. DiPrima, Elementary Differential Equations and Boundary Value Problems (Wiley, New York, 1977).

${ }^{17} \mathrm{R}$. Aris, Vectors, Tensors and the Basic Equations of Fluid Mechanics
} (Dover, New York, 1989). 